\title{
Utilizing diffractions in wavefront tomography
}

\author{
Alexander Bauer*, Benjamin Schwarz ${ }^{\dagger}$, and Dirk Gajewski* \\ *Institute of Geophysics \\ University of Hamburg \\ Bundesstraße 55 \\ D-20146 Hamburg (Germany) \\ ${ }^{\dagger}$ Department of Earth Sciences \\ University of Oxford \\ South Parks Road \\ Oxford OX1 3AN(United Kingdom)
}

(November 11, 2016)

Running head: Diffractions in wavefront tomography

\begin{abstract}
Wavefront tomography is known to be an efficient and stable approach for velocity inversion, which does not require accurate starting models and does not interact directly with the prestack data. Instead, the original data is transformed to physically meaningful wavefront attribute fields. These can be automatically estimated using local coherence analysis by means of the commonreflection-surface (CRS) stack, which has been shown to be a powerful tool for data analysis and enhancement. In addition, the zero-offset wavefront attributes acquired during the CRS stack can be used for sophisticated subsequent processes such as wavefield characterization and separation. Whereas in previous works, wavefront tomography has been applied mainly to reflection data, resulting in smooth velocity models suitable for migration of targets with moderately complex
\end{abstract}


overburden, this work has the emphasis on utilizing the diffracted contributions in the data for velocity inversion. By means of simple synthetic examples we demonstrate the potential of diffractions for velocity inversion. On industrial field data we suggest a joint inversion based on reflected and diffracted contributions of the measured wavefield, which confirms the general finding that diffraction-based wavefront tomography can help to increase the resolution of the velocity models. Concluding this work, we compare the quality of a reverse-time migrated result using the estimated velocity model with the result based on the inversion of reflections, which reveals an improved imaging potential for a complex salt geometry. 


\section{INTRODUCTION}

The estimation of seismic velocities plays a central role in physically characterizing the Earth's interior on scales ranging from the exploration of hydrocarbon reservoirs in local regimes of the crust up to global investigations of the Earth's deeper mantle and core (e.g., Romanowicz, 2003). Besides their potential to answer fundamental geodynamical questions, seismic velocity models are also needed for the migration of recorded seismic data to the depth domain, which leads to structurally rich images of back-scattering impedance contrasts in the subsurface (Claerbout, 1970). In recent years, the direct inversion of full broadband waveforms has become the primary tool for inferring the velocity structure with the highest possible resolution (Tarantola, 1984). Although being based on principles, which were already defined in the eighties, full-waveform inversion (FWI) is still computationally highly demanding and in 3D can only be afforded by processers with access to high-performance computing facilities (e.g., Virieux and Operto, 2009). In addition, current implementations still mostly have to rely on large-offset acquisitions of diving waves with low frequencies or reasonably accurate starting velocity models to ensure stable convergence to the global misfit minimum (Virieux and Operto, 2009). The established process of depth migration, in turn, is designed to provide structural images of high resolution by focusing the back-scattered, reflected and diffracted contributions of the excited wavefield even at principally small sourcereceiver offsets. In contrast to full-waveform inversion, imaging performs well for comparably smooth models with less detail in the velocity structure (e.g., Yilmaz, 2001).

While full-waveform inversion and depth migration in principle do not require a dense distribution of sources and receivers, Billette and Lambaré (1998) and Duveneck (2004) have introduced tomographic inversion techniques that exploit redundancy in the data, which is common in modern seismic acquisitions. These techniques can be formulated either in the pre- or in the poststack 
domain and rely on the local coherence of the recorded wavefield. Prestack stereotomography incorporates the wavefield's traveltimes, and, in addition to conventional traveltime tomography, its local slopes in the inversion (Billette and Lambaré, 1998). While this local description, in principle, allows for a high resolution in the inverted models, the poststack approach offers the benefits of a decreased noise level and data reduction, which can help to speed up and stabilize the process (Lavaud et al., 2004). The multi-dimensional common-reflection-surface (CRS) stack (Jäger et al., 2001) is a tool, which not only aims at optimally utilizing the redundancy in the data, but also automatically extracts valuable first- and second-order wavefront attributes, which can be used directly for automatic velocity inversion (Duveneck, 2004). In contrast to prestack stereotomography, this CRS-based wavefront tomography relies on a second-order symmetry of the kinematics of the wavefield in the common-midpoint (CMP) gather, which can be expressed by an auxiliary one-way wave originating at the normal-incidence point (NIP) of the central ray (Hubral, 1983).

While previous applications had a strong emphasis on reflected events (Duveneck, 2004; Dümmong et al., 2008), we aim to take into account the diffractions in the data. Since diffractions are the seismic response of small-scale structures, they are crucial for high-resolution imaging of the subsurface (Klem-Musatov et al., 1994; Moser and Howard, 2008; Dell and Gajewski, 2011). Santos et al. (2012) propose to make use of diffractions in the framework of tomographic inversion based on common-focus-point operators (CFPOs). Recent works indicate that for diffractions, poststack attributes can be used to accurately describe the full kinematics of the prestack response (Schwarz et al., 2014a; Bauer et al., 2016), which suggests a potential for increased resolution and stability of wavefront tomography. In this work, we make use of the non-Snell scattering contributions in the data for obtaining better-resolved velocity models than with conventional applications, which are generally reflection-based. Starting with simple synthetic examples, we find support of the recently formulated diffraction symmetry. Concluding this paper, we apply wavefront tomogra- 
phy to industrial field data acquired in the Eastern Mediterranean offshore Israel. We propose a joint inversion based on data points picked automatically at high-amplitude reflections as well as data points extracted from diffraction-only data. This promising approach reveals the potential of increased resolution of diffraction-based inversion for a complex salt geometry.

\section{COMMON-REFLECTION-SURFACE STACK}

Wavefront tomography is based on wavefront attributes that can be obtained by means of the zero-offset (ZO) common-reflection-surface (CRS) stack (Jäger et al., 2001), in which a zero-offset section is simulated in an automatic fashion. The ZO CRS stack is a multi-parameter stacking technique, which takes into account traces along a stacking surface in both offset and midpoint direction around a central midpoint $x_{0}$. Thus, the signal-to-noise ratio in the resulting zero-offset section can be significantly reduced without providing any prior model information. The 2D CRS stacking operator depends on three parameters, which can be expressed in terms of the geometrical wavefront attributes $\alpha_{0}, R_{N I P}$ and $R_{N}$ (Hubral, 1983). The parameter $\alpha_{0}$ describes the emergence angle, at which two ficticious waves emerge at the central midpoint $x_{0}$ on the recording surface. The attributes $R_{N I P}$ and $R_{N}$ describe the wavefront curvatures of these two ficticious waves, the NIP-wave and the normal wave, which are excited by a point source placed on the reflector's normal-incidence point (NIP) and an exploding reflector segment around the NIP, respectively. In the case of a diffraction, $R_{N I P}$ and $R_{N}$ coincide and the NIP-wavefront corresponds to the actual wavefront of the diffraction. This criterion can be utilized for the separation of reflections and diffractions in the resulting zero-offset sections (Dell and Gajewski, 2011). The hyperbolic zero-offset CRS stacking operator is given by (Jäger et al., 2001)

$$
t_{Z O}^{2}=\left(t_{0}+2 p_{0} \Delta x_{m}\right)^{2}+2 t_{0}\left(M_{N} \Delta x_{m}^{2}+M_{N I P} h^{2}\right)
$$


where $\Delta x_{m}=x_{m}-x_{0}$ is the displacement from the central midpoint $x_{0}, b$ denotes the half-offset and $t_{0}$ the traveltime of the central zero-offset ray emerging at $x_{0}$. The horizontal slowness of the normal ray can also be expressed in terms of the geometrical wavefront attributes,

$$
p_{0}=\frac{\sin \alpha_{0}}{v_{0}}
$$

where $v_{0}$ is the constant near-surface velocity. The second derivative of the traveltime in a CMPgather is then given by

$$
M_{N I P}=\frac{\cos ^{2} \alpha_{0}}{v_{0} R_{N I P}},
$$

and accordingly for $M_{N}$. Although the hyperbolic traveltime operator (1) is the most common one, different moveout descriptions, such as multifocusing (Gelchinsky et al., 1999), non-hyperbolic CRS (Fomel and Kazinnik, 2013) or implicit CRS (Schwarz et al., 2014b), can be used to obtain wavefront attributes. At each zero-offset sample $\left(x_{0}, t_{0}\right)$, values for the three wavefront attributes are determined by coherence analysis (usually, the semblance coefficient (Taner and Koehler, 1969) is used as a measure of coherence) using either a pragmatic approach and subsequent local optimization (Mann, 2002) or a global optimization scheme (Walda and Gajewski, 2015). Also conflicting dips can be considered by estimating more than one operator at each sample (Mann, 2002; Soleimani et al., 2009; Walda and Gajewski, 2015). Since the wavefront attributes obtained from the ZO CRS stack have a physical meaning, they can be exploited for further processing steps, such as diffraction separation (Dell and Gajewski, 2011), prestack data enhancement (Baykulov and Gajewski, 2009; Bauer et al., 2016) or the tomographic inversion revisited in this paper (Duveneck, 2004). 


\section{DIFFRACTION SYMMETRY}

Since Snell's law does not hold in the case of a point diffractor, diffraction raypaths are decoupled and zero-offset and finite-offset information (Zhang et al., 2001) is redundant for the corresponding traveltime response (Schwarz et al., 2014a; Bauer et al., 2016). As illustrated in Figure 1, the common-shot and common-receiver responses in a finite-offset measurement of a diffraction are technically identical to two independent zero-offset measurements carried out at $x_{0}^{s}$ and $x_{0}^{g}$. Based on the assumption of reciprocity, which is a model-independent universal principle valid for monotypic waves, it is possible to decompose any finite-offset diffraction operator into two independent zero-offset operators at a source $x_{0}^{s}$ and a receiver $x_{0}^{g}$ (Schwarz et al., 2014a; Bauer et al., 2016),

$$
t_{F O}=\frac{t_{Z O}\left(x_{0}^{s}, t_{0}^{s}, p_{0}^{s}, M_{N I P}^{s}\right)}{2}+\frac{t_{Z O}\left(x_{0}^{g}, t_{0}^{g}, p_{0}^{g}, M_{N I P}^{g}\right)}{2} .
$$

This traveltime decomposition principle is a fundamental property of diffractions and thus, Equation (4) holds independently of the choice of zero-offset operators. If, e.g., hyperbolic operators such as (1) are used during the zero-offset processing, the finite-offset diffraction operators composed according to (4) correspond to double-square-root operators (Yilmaz, 2001), in which the two square roots are approximated independently. Given the results from zero-offset processing are accurate, the decomposition principle is exact for diffractions in arbitrary media. Note that it is only valid for diffractions, because it is based on the assumption of decoupled up- and downgoing raypaths. Accordingly, Equation (4) does not apply in the reflection case.

By utilizing diffractions in the zero-offset-based poststack wavefront tomography we aim to make use of the fact that, unlike the reflection case, the full prestack diffraction response is encoded in its zero-offset measurements, which suggests a potential for improved lateral resolution of the velocity models. 


\section{WAVEFRONT TOMOGRAPHY}

Wavefront tomography, introduced by Duveneck (2004), is an efficient tomographic approach, which is entirely based on the zero-offset CRS attributes $p_{0}$ and $M_{N I P}$ and provides smooth 2D isotropic velocity models. Previous to the inversion, independent data points are picked in a datadriven way in the CRS semblance section by taking into account stack amplitudes and attribute values. During the tomographic inversion, rays are propagated through the model starting from the picked locations with the respective attribute values. The velocity model is updated according to the criterion that in a correct model, all considered NIP-waves focus at zero traveltime.

\section{Input data and model}

The input for the inversion are automatically picked data points of the CRS results, which are selected based on their coherence and a number of predefined constraints. Each pick is defined by its location on the recording surface $x_{0}$, its zero-offset traveltime $t_{0}$ and the wavefront attributes $p_{0}$ and $M_{N I P}$ associated with the picked event (see Figure 2). Instead of $t_{0}$, wavefront tomography considers the one-way zero-offset traveltime

$$
T_{0}=\frac{t_{0}}{2}
$$

Thus, the input data for the inversion algorithm is given by

$$
\left(x_{0}, T_{0}, p_{0}, M_{N I P}\right)_{i} \quad \text { with } \quad i=1, \ldots, n_{\text {picks }},
$$

where $n_{\text {picks }}$ is the total number of automatically picked data points. Each point $i$ can be associated with an imaginary normal-incidence point NIP* in the subsurface, which for a constant initial model can be obtained by straight-ray projection into the optical image space (see Figure 2).

Only in a correct velocity model $v(x, z)$, NIP* coincides with the true NIP. In theory, checking 
if the back-propagated NIP-waves starting from $x_{0}^{i}$ with $p_{0}^{i}$ for all picks focus at $T_{0}^{i}=0$ is a natural condition for the inversion. However, we have to take into account that the data might be affected by errors in order to ensure stability. Therefore, at every NIP* associated to one data point $i$, a ray is started in the subsurface by dynamic ray tracing (Duveneck, 2004). During this process, the true subsurface positions $(x, z)_{i}$ and the takeoff angles $\theta_{i}$ of the rays must be considered a part of the model, because they are unknown. In order to find the optimum velocity model, wavefront tomography minimizes the misfit between measured and modeled values $\left(x_{0}, T_{0}, p_{0}, M_{N I P}\right)_{i}$. Billette and Lambaré (1998) used a similar approach for stereotomography, which is a tomographic inversion method working in the prestack domain.

For the description of the smooth velocity model $v(x, z)$, wavefront tomography uses 2D Bsplines,

$$
v(x, z)=\sum_{j=1}^{n_{x}} \sum_{k=1}^{n_{z}} v_{j k} \beta_{j}(x) \beta_{k}(z),
$$

where $n_{x}$ and $n_{z}$ are the total number of knots in $x$ and $z$-direction, respectively. Accordingly, the model is defined by the B-spline coefficients $v_{j k}$ and the coordinates and ray takeoff angles related to the data points,

$$
\begin{aligned}
(x, z, \theta)_{i} & \text { with } \quad i=1, \ldots, n_{\text {picks }}, \\
v_{j k} & \text { with } \quad(j, k)=(1,1), \ldots,\left(n_{x}, n_{z}\right) .
\end{aligned}
$$

In order to obtain the desired optimum velocity model, the inverse problem has to be solved iteratively. This process will be described in the following section.

\section{Solution of the inverse problem}

In order to solve the inverse problem, the algorithm has to find a model vector $\mathbf{m}$, which minimizes the misfit between a data vector $\mathbf{d}$ and the corresponding modeled values $\mathbf{d}_{\text {mod }}=\mathbf{f}(\mathbf{m})$ (Duveneck, 
2004), where the model vector $\mathbf{m}$ contains all model components (8), the data vector $\mathbf{d}$ consists of all picked data points (6) and $\mathbf{f}$ is a nonlinear operator, which, in the case of wavefront tomography, corresponds to the dynamic ray tracing carried out in the model. The presented inversion algorithm uses the least-squares norm (Paige and Saunders, 1982; Tarantola, 2005) as a measure of misfit and tries to minimize the objective function given by

$$
\Psi(\mathbf{m})=\frac{1}{2}\|\mathbf{d}-\mathbf{f}(\mathbf{m})\|_{D}^{2}=\frac{1}{2} \Delta \mathbf{d}^{T}(\mathbf{m}) \mathbf{C}_{D}^{-1} \Delta \mathbf{d}(\mathbf{m})
$$

where $\Delta \mathbf{d}(\mathbf{m})=\mathbf{d}-\mathbf{f}(\mathbf{m})$. The matrix $\mathbf{C}_{D}^{-1}$ is symmetric and positive definite and weights the different data components during the calculation of $\Psi$. Due to the nonlinearity of the operator $\mathrm{f}$, the inverse problem is solved iteratively by locally linearizing $f(\mathbf{m})$ around the current model vector. For this, the Fréchet derivatives of $\mathbf{f}$ are needed, which can be calculated during forward modeling (Farra and Madariaga, 1987). In order to facilitate a stable inversion, the requirement of a smooth velocity model is implemented as an additional constraint by minimizing its second derivatives. This results in an additional term in the objective function,

$$
\Psi(\mathbf{m})=\frac{1}{2} \Delta \mathbf{d}^{T}(\mathbf{m}) \mathbf{C}_{D}^{-1} \Delta \mathbf{d}(\mathbf{m})+\frac{1}{2} \epsilon^{\prime \prime} \mathbf{m}_{(v)}^{T} \mathbf{D}^{\prime \prime} \mathbf{m}_{(v)}
$$

where $\mathbf{m}_{(v)}$ is the part of the model vector $\mathbf{m}$ containing the velocity coefficients and $\epsilon^{\prime \prime}$ is a weighting factor, which balances the contributions of the data misfit term and the regularization term to the cost function. The additional term contains the integral

$$
\mathbf{m}_{(v)}^{T} \mathbf{D}^{\prime \prime} \mathbf{m}_{(v)}=\iint\left[\epsilon_{x x}\left(\frac{\partial^{2} v(x, z)}{\partial x^{2}}\right)^{2}+\epsilon_{z z}\left(\frac{\partial^{2} v(x, z)}{\partial z^{2}}\right)^{2}\right] \mathrm{d} x \mathrm{~d} z
$$

where the factors $\epsilon_{x x}$ and $\epsilon_{z z}$ are used for weighting the contributions of the corresponding second derivatives. The additional term is added in order to ensure that the matrix $\mathbf{D}^{\prime \prime}$ is positive definite. For the explanation of the matrix $\mathbf{D}^{\prime \prime}$ and a more detailed description of the solution of the inverse problem we refer to the work of Duveneck (2004). Optionally, the weighting factors $\epsilon_{x x}$ and $\epsilon_{z z}$ 
may be decreased successively after each iteration by multiplying them with a factor $\eta$ given by

$$
\eta=\sqrt{\frac{\Psi_{l}}{\Psi_{l-1}}},
$$

where $l$ is the current iteration and $\Psi_{l}$ and $\Psi_{l-1}$ the corresponding values of the objective function (10) after the current and the previous iteration, respectively.

After setting up an initial velocity model, defined by a constant vertical velocity gradient or a given starting model, kinematic ray tracing for each data point $\left(x_{0}, T_{0}, p_{0}, M_{N I P}\right)_{i}$ yields the corresponding coordinates $(x, z, \theta)_{i}$ in the model. As a next step, the algorithm performs dynamic ray tracing in the upward direction until the rays, which are started at $(x, z)_{i}$ with the angles $\theta_{i}$, reach the measurement surface. The updated model for the $l$-th iteration is then given by $\mathbf{m}_{l+1}=\mathbf{m}_{l}+\lambda \Delta \mathbf{m}$, where $0<\lambda \leq 1$ (for more details, see Duveneck, 2004). In this model, new data values are obtained from dynamic ray tracing and the objective function (10) is calculated. If its value decreases with respect to the previous iteration, the next iteration is started. Otherwise, the factor $\lambda$ is decreased and the objective function is recalculated. If a minimum of the objective function is reached, the calculation is stopped.

\section{SIMPLE DIFFRACTION DATA}

In previous studies (Duveneck, 2004; Meier, 2007; Dümmong et al., 2008), wavefront tomography has been applied mainly based on input data points picked on high-amplitude reflections. However, diffraction events possess properties, which make them an interesting target for wavefront tomography. Whereas in the case of a reflection, the NIP-wave emerges from a hypothetical point source placed on the reflector's normal-incidence point, in the case of a diffraction, the NIP-wave is excited by the diffractor itself and therefore, $p_{0}$ and $M_{N I P}$ describe the actual wavefront of the diffraction. Furthermore, diffractions imply better illumination than reflections, because a point 
diffractor scatters an incoming wave into all directions and thus, contributions stemming from the same diffractor can be recorded within a large part of the recording surface. This means that the rays corresponding to just one diffraction may already travel through a significant part of the model. In addition, the proximity of the starting coordinates of all rays connected to one diffraction serves as a strong quality criterion for the inverted model.

In order to illustrate the procedure, we applied wavefront tomography to a simple dataset containing one diffraction based on a model with a constant vertical velocity gradient of $0.5 \mathrm{~s}^{-1}$. The results are displayed in Figure 3, where Figure 3a shows the data points, which were picked automatically in the zero-offset CRS semblance section. As expected, all 108 picks lie on the diffraction hyperbola. Figure $3 \mathrm{~b}$ shows the initial velocity model for the tomographic inversion, which merely consists of the near-surface velocity $v_{0}$ and no initial vertical velocity gradient. The black asterisks indicate the starting locations of the rays. In a correct velocity model, these locations should coincide for all rays stemming from the same diffractor. In this example, we used $18 \times 18$ B-spline knots with a spacing of $250 \mathrm{~m}$ in $x$-direction and $100 \mathrm{~m}$ in $z$-direction for the tomographic inversion. Figure $3 \mathrm{c}$ shows the inverted model after 13 iterations, the final starting coordinates of the rays and the corresponding ray trajectories. A comparison of the inverted model with the correct one displayed in Figure $3 \mathrm{~d}$ reveals a good agreement in those parts of the model illuminated by the rays. In addition, although all picked data points are treated independently by the algorithm, the final starting locations of the rays are confined to the same depth point, which coincides with the actual position of the diffractor indicated by the black dot in the correct model. Although only a single diffractor was used, the gradient in the illuminated area is already very well reconstructed. By increasing the number of diffractors this result will further improve, which is demonstrated in the next example.

We applied the same workflow to a dataset based on the same background model, but con- 
taining a larger number of diffractors at different locations in the subsurface. Figure 4 shows the results of this application. In Figure 4a, the asterisks indicate the 992 automatically picked data points in the zero-offset CRS semblance section. For the tomographic inversion, we used 26 Bspline knots in $x$-direction and 21 knots in $z$-direction with a spacing of $200 \mathrm{~m}$ each. As in the previous example, the initial model consisted of the constant near-surface velocity $v_{0}$, as displayed in Figure 4b, where the starting locations of the rays again indicate the wrong velocity model. The result of the inversion is displayed in Figure $4 \mathrm{c}$ including the final starting coordinates of the rays. In this example, we stopped the calculation after 50 iterations, because the value of the objective function did not change significantly anymore. The ray trajectories are not displayed in this case for a better visualization of the result. In the inverted model, the ray starting locations are, except for a few outliers, confined nicely to eight distinct depth points, although every data point is treated independently by the inversion. The correct model shown in Figure $4 \mathrm{~d}$ reveals that the inverted depth points coincide very well with the true diffractor positions. Note that even the two diffractors lying close together on the upper left side of the images are distinguishable. Since almost the whole model space is covered by rays, also the background velocity gradient could be well recovered during the inversion.

\section{FIELD DATA}

Finally, we applied the same workflow to a marine field dataset recorded by TGS in the Levantine Basin, which is located in the Eastern Mediterranean offshore Israel. The profile considered in this work is characterized by pronounced salt-roller structures in the left part (Hübscher and Netzeband, 2007). In previous studies, Meier (2007) and Dümmong et al. (2008) applied both wavefront tomography and prestack stereotomography (Billette and Lambaré, 1998) to various lines acquired in the same region. However, their results were based on data points mainly picked 
on high-amplitude reflections. In order to increase the resolution of the resulting velocity models, we propose to take into account diffractions, which usually have lower amplitudes than reflections.

Figure $5 \mathrm{a}$ shows the zero-offset stack of the dataset under investigation. It was obtained with non-hyperbolic CRS (Fomel and Kazinnik, 2013) using a global optimization algorithm accounting for a maximum of 17 conflicting events at one sample (Walda and Gajewski, 2015). In order to be able to automatically pick low-amplitude events in the data, we carried out a zero-offset diffraction separation, which is based on the assumption that the second-order attributes $M_{N I P}$ and $M_{N}$ coincide in the case of a diffraction. Their similarity is evaluated at each zero-offset sample by a weight function (Dell and Gajewski, 2011) of the form

$$
w\left(x_{0}, t_{0}\right)=\exp \left(-\frac{\left|M_{N}-M_{N I P}\right|}{\left|M_{N}+M_{N I P}\right|}\right)
$$

which reaches its maximum 1 if $M_{N I P}=M_{N}$. In this case, we defined a threshold of $w=0.9$ and only stacked the events with a larger value of $w$, while the remaining events were neglected. The resulting diffraction-only stack is displayed in Figure 5b. The result reveals numerous lowamplitude events, which are masked by reflections in the full zero-offset stack.

For the automatic picking of events, we propose the following strategy: first, picking of highamplitude events in the full zero-offset semblance section. Afterwards, picking of low-amplitude events in the diffraction-only semblance section using a lower semblance threshold. Note that this picking process is fully automatic and only relies on the semblance and the stack section, which both offer the advantage of an increased signal-to-noise ratio. In order to get rid of data points corresponding to multiple reflections, we plotted the two-way time $t_{0}$ of all picks against the corresponding $M_{N I P}$ and sorted out all data points not following the general trend. Thus, we obtained a total number of 11955 data points, of which 6430 were extracted from the full semblance section and 5525 from the diffraction-only data. Finally, we carried out a joint tomographic inversion 
using all picked data points together as input. Figure 6a shows all data points in the full zero-offset semblance section.

For the inversion, we used a grid of $136 \times 41 \mathrm{~B}$-spline knots with a spacing of $200 \mathrm{~m}$ in $x$ and $100 \mathrm{~m}$ in $z$-direction. We assumed to have no a priori information of the velocity in the subsurface. Accordingly, the initial model solely consisted of the near-surface velocity $v_{0}=1480 \mathrm{~ms}^{-1}$. It is displayed in Figure $6 \mathrm{~b}$ together with the initial ray starting locations associated to each data point. During the inversion, we constrained the velocity within the water column to $v_{0}$. The inverted model is displayed in Figure 6c overlain with the final ray starting locations associated to each data point. Figure $7 \mathrm{~b}$ shows a clean version of the model obtained by joint inversion of diffractions and reflections, whereas Figure 7a shows a velocity model resulting from an inversion of the high-amplitude reflections only (6430 data points). Both results were obtained after 6 inversion runs each, during which the regularization weights $\epsilon_{x x}$ and $\epsilon_{z z}$ were systematically decreased in order to increase the model resolution. Whereas the first inversion run was started with the initial model displayed in Figure 6b, the subsequent runs were started with the final result of the respective previous run. The total number of iterations was 227 (joint inversion) and 239 (reflection-only inversion). The final model of the joint inversion (Figure 7b) reveals a highvelocity zone, which is more prominent to the left of the profile. This is in agreement with the salt geometry known to be present in the region (see also Hübscher and Netzeband, 2007; Dümmong et al., 2008). Compared to the results of previous studies (Meier, 2007; Dümmong et al., 2008), our result confirms the general velocity distribution. A comparison of the velocity models obtained by joint inversion (Figure 7b) and reflection-only inversion (Figure 7a) reveals that the incorporation of low-amplitude diffractions enhances the resolution of the velocity model and even reveals new features, particularly on the right side of the profile.

In order to examine the validity of the velocity model from joint inversion (Figure 7b), we 
used it as input for a reverse-time migration (RTM) algorithm (e.g., Baysal et al., 1983; Schuster, 2002). The used RTM implementation is based on a deconvolution imaging condition in the time domain (Valenciano and Biondi, 2002). The result is displayed in Figure 8 overlain with the velocity model. It shows that the salt-roller structures could be imaged clearly along the whole profile, even at the steep flanks. Also the reflections from the bottom of the salt are clearly visible. The general velocity structure is in good agreement with the salt geometry imaged by the RTM. For comparison we applied the RTM algorithm again using the reflection-based velocity model (Figure 7a). Figure 9 shows excerpts of the migrated images using the reflection-only velocity model (Figure 9a) and the velocity model based on joint inversion (Figure 9b) in order to compare the prominent salt-roller structures in the dataset. The salt geometry appears better resolved along the whole profile in the RTM image based on the joint inversion and particularly the rugged structures on the right side of the profile, indicated by strong diffractions in the CRS stacks (see Figure 5), are imaged more clearly and with larger amplitudes. For further examination of the quality of the migration results, Figure 10 shows common-image-gathers (CIGs) taken from four different lateral positions along the profile with a maximum offset of $2 \mathrm{~km}$ each. The CIGs taken from the RTM results based on the joint inversion (Figure 10b) generally appear flatter than the ones taken from the reflection-based RTM result (Figure 10a), indicating a better quality of the depth migration. Particularly large differences are visible for the top-of-salt events at about $2.2 \mathrm{~km}$ depth, which confirms the previously made observation that taking diffractions into account during velocity inversion not only improves the resolution of the velocity model, but also enhances the resulting depth-migrated image. 


\section{DISCUSSION}

The results presented in this paper confirm that poststack wavefront tomography is an efficient and stable tool to obtain highly resolved subsurface velocity models, which does not require any a priori velocity information except the usually well-known near-surface velocity. Furthermore, unlike other inversion techniques such as prestack stereotomography (Billette and Lambaré, 1998) or full-waveform inversion (Virieux and Operto, 2009), CRS-based wavefront tomography does not interact with the full prestack data volume, but relies on wavefront attributes defined in the poststack domain. These wavefront attributes can be estimated e.g. by the zero-offset CRS stack or any other operator of the CRS family using local coherence analysis, which allows automatic picking of data points for the tomographic inversion. While this implies a certain robustness of the method with respect to, e.g., random noise, errors made in the coherence and attribute estimation may have significant impact on the quality of the inversion. Therefore, further improvement of the CRS stack, by utilizing global optimization strategies (e.g., Walda and Gajewski, 2015) or by incorporating more accurate moveout approximations that are better suited for diffractions (e.g., Schwarz et al., 2014b), as well as the design of more sophisticated diffraction filters is of central importance to improve the suggested approach to poststack wavefront tomography.

While previous applications were mainly based on high-amplitude reflections in the data, we propose utilizing diffractions for improving the resolution of the velocity models. As the simple synthetic examples show, diffractions can contribute to velocity inversion due to their better illumination of the subsurface. In addition, they provide a new criterion for the quality of the inversion results, because in a correct model, all contributions belonging to one diffractor should focus at the same subsurface position. While we treated each data point independently in this work, in future work we aim to incorporate the focusing criterion for diffractions as a constraint 
into the inversion algorithm.

On complex industrial field data, we suggest independent extraction of reflected and diffracted events from the data and a joint inversion using all data points together. In this fashion, the general velocity distribution may be steered by the high-amplitude reflections, whereas the diffracted contributions serve to enhance the resolution of the velocity model. While this higher resolution helps to improve depth-migration results, the resulting velocity models do not feature a resolution comparable to results of methods like full-waveform inversion, but they may serve as high-quality starting models for these sophisticated inversion techniques improving cycle-skip issues.

Since diffractions, in contrast to reflections, from a kinematic point of view are a one-way process, their moveout can be described entirely in the zero-offset section (Schwarz et al., 2014a; Bauer et al., 2016), which supports the nature of our zero-offset-based approach. Wavefront tomography is also applicable for joint localization and velocity inversion in a passive seismic setting. First promising results have been published recently by Schwarz et al. (2016).

\section{CONCLUSIONS}

In this paper, we revisited the largely automatic process of wavefront tomography as an efficient tool to infer the seismic velocity structure of the subsurface, which does not require a priori velocity information. With special emphasis on non-Snell scattering, we illustrated by means of simple synthetic examples that the use of diffracted contributions can enhance the resolution of the inverted models. On industrial field data, the joint inversion of reflected and diffracted contributions led to a reliable detection of the salt and to an increased resolution of the velocity model compared to the conventional result gained by favoring reflections. Results of reverse-time migration based on the improved velocity model revealed a better imaging of the complex salt geometry 
in the depth domain, which is confirmed by flatter common-image gathers.

\section{ACKNOWLEDGMENTS}

The contributions of Ekkehart Teßmer in the RTM processing are highly appreciated. We thank Manuel Lotze and Tobias Werner for their valuable contributions in the course of this project and Jan Walda for the generation of wavefront attributes for the field data. This work was kindly supported by the sponsors of the Wave Inversion Technology (WIT) Consortium, Hamburg, Germany, and is part of the project Imaging steep structures with diffractions funded by the German Federal Ministry of Economic Affairs and Energy (BMWi 0325363C). We thank the applied seismics group at the University of Hamburg for continuous discussion and TGS for providing the

marine field data. Seismic Unix was used for data processing, for creating the simple synthetic datasets and for the generation of figures. 


\section{REFERENCES}

Bauer, A., B. Schwarz, and D. Gajewski, 2016, Enhancement of prestack diffraction data and attributes using a traveltime decomposition approach: Studia Geophysica et Geodaetica, 60, 471486.

Baykulov, M., and D. Gajewski, 2009, Prestack seismic data enhancement with partial commonreflection-surface (CRS) stack: Geophysics, 74, V49-V58.

Baysal, E., D. D. Kosloff, and J. W. Sherwood, 1983, Reverse time migration: Geophysics, 48, 1514-1524.

Billette, F., and G. Lambaré, 1998, Velocity macro-model estimation from seismic reflection data by stereotomography: Geophysical Journal International, 135, 671-690.

Claerbout, J., 1970, Coarse grid calculations of waves in inhomogeneous media with application to delineation of complicated seismic structure: Geophysics, 35, 407-418.

Dell, S., and D. Gajewski, 2011, Common-reflection-surface-based workflow for diffraction imaging: Geophysics, 76, S187-S195.

Dümmong, S., K. Meier, D. Gajewski, and C. Hübscher, 2008, Comparison of prestack stereotomography and NIP wave tomography for velocity model building: Instances from the Messinian evaporites: Geophysics, 73, VE291-VE302.

Duveneck, E., 2004, Velocity model estimation with data-derived wavefront attributes: Geophysics, 69, 265-274.

Farra, V., and R. Madariaga, 1987, Seismic waveform modeling in heterogeneous media by ray perturbation theory: Journal of Geophysical Research: Solid Earth (1978-2012), 92, 2697-2712.

Fomel, S., and R. Kazinnik, 2013, Non-hyperbolic common reflection surface: Geophysical Prospecting, 61, 21-27.

Gelchinsky, B., A. Berkovitch, and S. Keydar, 1999, Multifocusing homeomorphic imaging: Part 
1. basic concepts and formulas: Journal of Applied Geophysics, 42, 229-242.

Hubral, P., 1983, Computing true amplitude reflections in a laterally inhomogeneous earth: Geophysics, 48, 1051-1062.

Hübscher, C., and G. Netzeband, 2007, Evolution of a young salt giant: The example of the messinian evaporites in the levantine basin, in The mechanical behaviour of salt: Taylor \& Francis, 175-184.

Jäger, R., J. Mann, G. Höcht, and P. Hubral, 2001, Common-reflection-surface stack: Image and attributes: Geophysics, 66, 97-109.

Klem-Musatov, K., F. Hron, and L. Lines, 1994, Theory of seismic diffractions: Society of Exploration Geophysicists.

Lavaud, B., R. Baina, and E. Landa, 2004, Automatic robust velocity estimation by poststack stereotomography, in SEG Technical Program Expanded Abstracts 2004: Society of Exploration Geophysicists, 2351-2354.

Mann, J., 2002, Extensions and applications of the common-reflection-surface stack method: $\mathrm{PhD}$ thesis, University of Karlsruhe.

Meier, K., 2007, Velocity model building: A comparison between prestack stereotomography and nip-wave tomography: diploma thesis, University of Hamburg.

Moser, T., and C. Howard, 2008, Diffraction imaging in depth: Geophysical Prospecting, 56, $627-641$.

Paige, C. C., and M. A. Saunders, 1982, LSQR: An algorithm for sparse linear equations and sparse least squares: ACM Transactions on Mathematical Software (TOMS), 8, 43-71.

Romanowicz, B., 2003, Global mantle tomography: progress status in the past 10 years: Annual Review of Earth and Planetary Sciences, 31, 303-328.

Santos, L. A., W. J. Mansur, and G. A. McMechan, 2012, Tomography of diffraction-based focusing 
operators: Geophysics, 77, R217-R225.

Schuster, G. T., 2002, Reverse-time migration = generalized diffraction stack migration, in SEG Technical Program Expanded Abstracts 2002: Society of Exploration Geophysicists, 12801283.

Schwarz, B., A. Bauer, and D. Gajewski, 2016, Passive seismic source localization via commonreflection-surface attributes: Studia Geophysica et Geodaetica, 60, 531-546.

Schwarz, B., C. Vanelle, and D. Gajewski, 2014a, From zero-offset to common-offset with diffractions: Presented at the 76th EAGE Conference and Exhibition - Workshops.

Schwarz, B., C. Vanelle, D. Gajewski, and B. Kashtan, 2014b, Curvatures and inhomogeneities: an improved common-reflection-surface approach: Geophysics, 79, S231-S240.

Soleimani, M., I. Piruz, J. Mann, and P. Hubral, 2009, Solving the problem of conflicting dips in common-reflection-surface (CRS) stack: Presented at the 1st International Petroleum Conference \& Exhibition.

Taner, M. T., and F. Koehler, 1969, Velocity spectra - digital computer derivation and applications of velocity functions: Geophysics, 34, 859-881.

Tarantola, A., 1984, Inversion of seismic reflection data in the acoustic approximation: Geophysics, 49, 1259-1266.

---, 2005, Inverse problem theory and methods for model parameter estimation: SIAM.

Valenciano, A. A., and B. Biondi, 2002, Deconvolution imaging condition for reverse-time migration: Stanford Exploration Project, Report, 112, 83-96.

Virieux, J., and S. Operto, 2009, An overview of full-waveform inversion in exploration geophysics: Geophysics, 74, WCC1-WCC26.

Walda, J., and D. Gajewski, 2015, Common-reflection-surface stack improvement by differential evolution and conflicting dip processing, in SEG Technical Program Expanded Abstracts 2015: 
Society of Exploration Geophysicists, 3842-3847.

Yilmaz, Ö., 2001, Seismic data analysis: Society of Exploration Geophysicists, Tulsa, 1.

Zhang, Y., S. Bergler, and P. Hubral, 2001, Common-reflection-surface (CRS) stack for common offset: Geophysical Prospecting, 49, 709-718. 


\section{LIST OF FIGURES}

1 A finite-offset diffraction setting can be described exactly by two independent zero-offset measurements at a source $x_{0}^{s}$ and a receiver $x_{0}^{g}$.

2 The wavefront attributes of the NIP-wave are determined in the so-called optical image space (black) by assuming a medium with constant velocity $v_{0}$, in which the location NIP* is found by straight-ray projection. By means of wavefront tomography we aim to determine the true velocity model $v(x, z)$ in order to find the NIP-wave's true source location (gray).

3 Results for simple diffraction data containing one diffractor. (a) All 108 automatically picked data points plotted into the zero-offset semblance section. (b) The constant initial model for the inversion containing the initial ray starting locations. (c) The inverted model with the final ray starting locations and the final ray trajectories corresponding to each data point. (d) The correct velocity model with the position of the diffractor indicated by a black dot.

4 Results for simple diffraction data containing multiple diffractors. (a) All 992 automat-

ically picked data points plotted into the zero-offset semblance section. (b) The constant initial model for the inversion containing the initial ray starting locations. (c) The inverted model with the final ray starting locations. (d) The correct velocity model with the position of the diffractor indicated by a black dot.

5 (a) Zero-offset CRS stack of the investigated marine field data. (b) ZO CRS stack after diffraction separation.

6 Field data results. (a) All 11955 automatically picked data points plotted into the zerooffset semblance section. (b) The constant initial model for the inversion containing the initial ray starting locations. (c) Resulting model of the joint inversion using both reflection and diffraction picks overlain with the final ray starting locations.

$7 \quad$ Velocity models obtained by (a) inversion of only reflections and (b) joint inversion of 
reflections and diffractions.

8 Reverse-time depth-migrated image of the marine field data overlain with the final velocity model obtained from joint inversion of reflections and diffractions.

9 Excerpts of the reverse-time-migrated images obtained with (a) the reflection-based velocity model and (b) the velocity model from joint inversion of diffractions and reflections.

10 Common-image-gathers (CIGs) of reverse-time-migrated data obtained with (a) the reflection-based velocity model and (b) the velocity model from joint inversion of diffractions and reflections for four different midpoint locations with a maximum offset of $2 \mathrm{~km}$ each. 


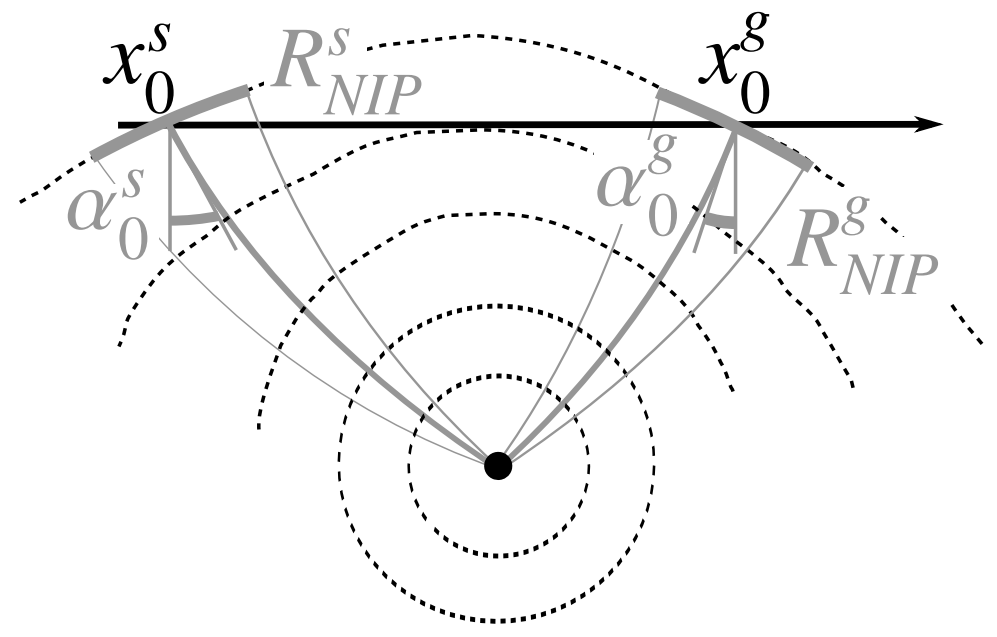

Figure 1: A finite-offset diffraction setting can be described exactly by two independent zero-offset measurements at a source $x_{0}^{s}$ and a receiver $x_{0}^{g}$.

Bauer, Schwarz \& Gajewski - 


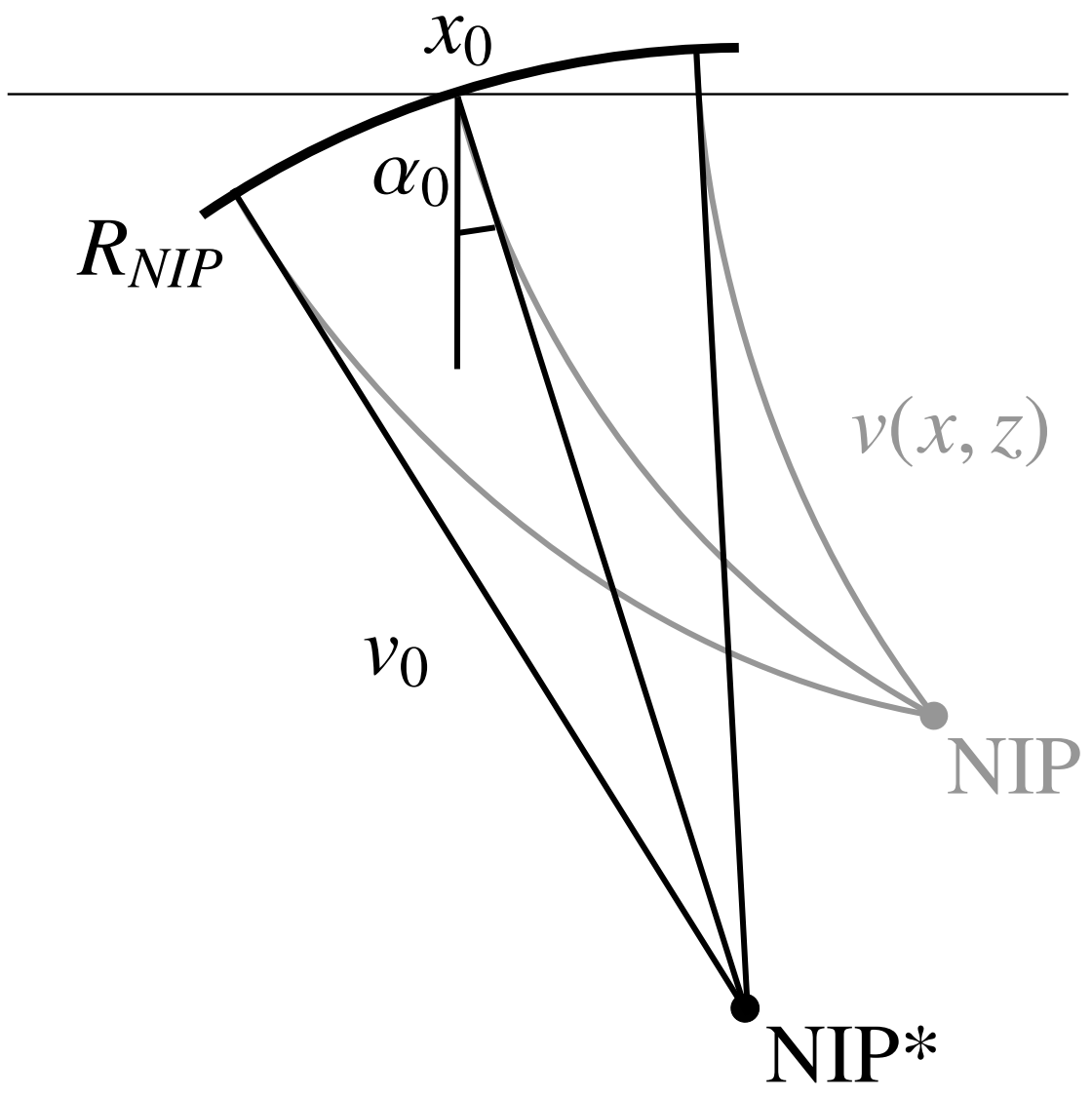

Figure 2: The wavefront attributes of the NIP-wave are determined in the so-called optical image space (black) by assuming a medium with constant velocity $v_{0}$, in which the location NIP* is found by straight-ray projection. By means of wavefront tomography we aim to determine the true velocity model $v(x, z)$ in order to find the NIP-wave's true source location (gray).

Bauer, Schwarz \& Gajewski - 


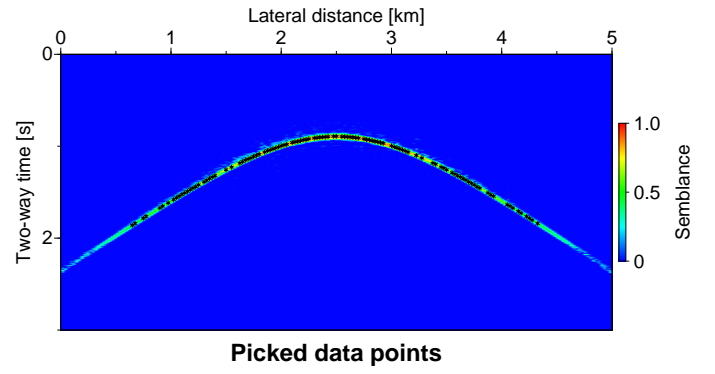

(a)

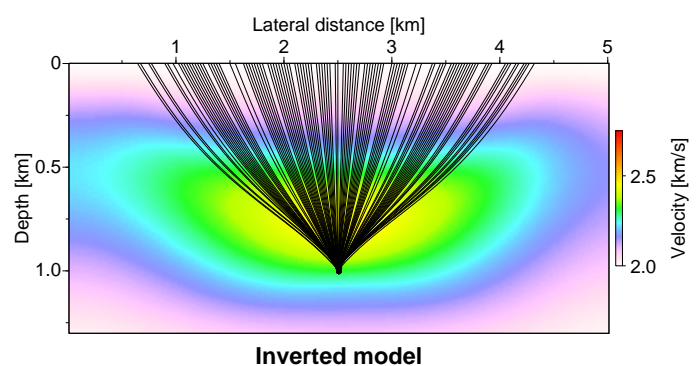

(c)

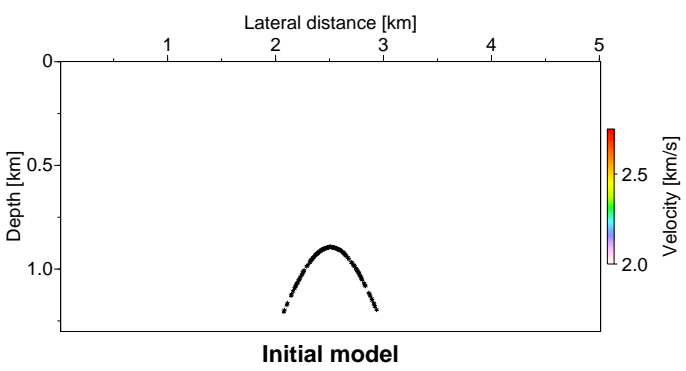

(b)

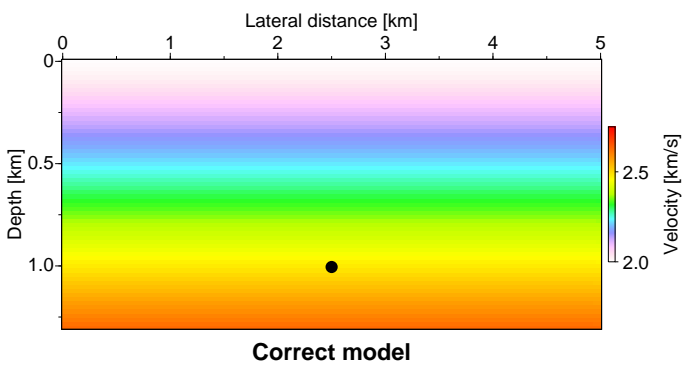

(d)

Figure 3: Results for simple diffraction data containing one diffractor. (a) All 108 automatically picked data points plotted into the zero-offset semblance section. (b) The constant initial model for the inversion containing the initial ray starting locations. (c) The inverted model with the final ray starting locations and the final ray trajectories corresponding to each data point. (d) The correct velocity model with the position of the diffractor indicated by a black dot.

\section{Bauer, Schwarz \& Gajewski -}




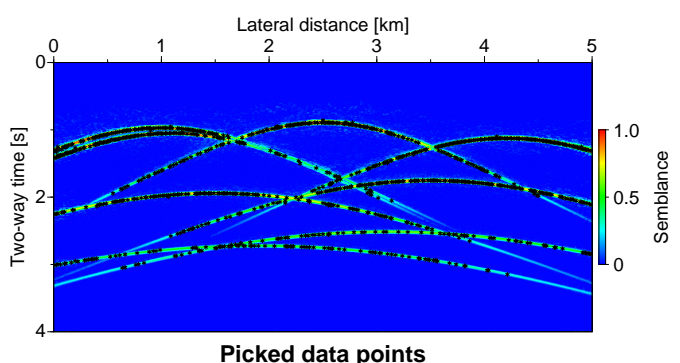

(a)

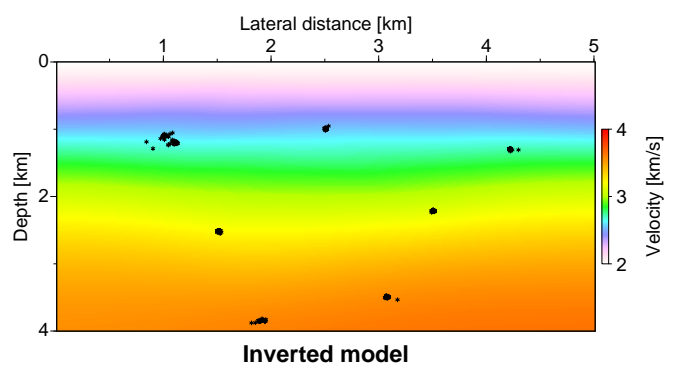

(c)

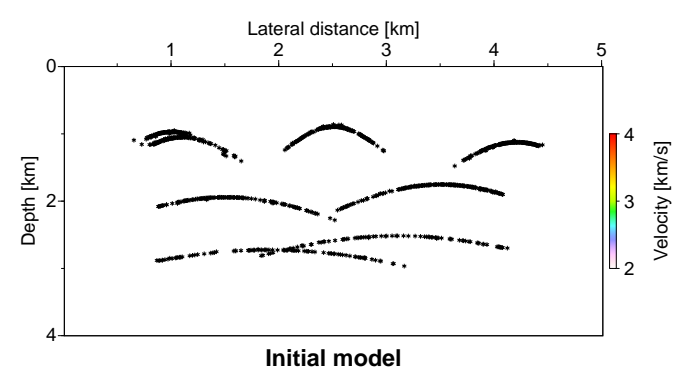

(b)

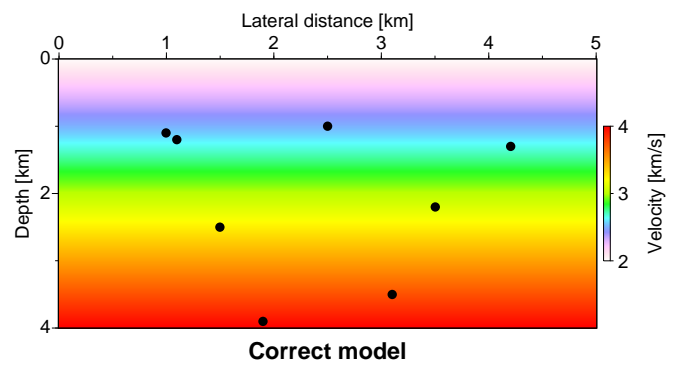

(d)

Figure 4: Results for simple diffraction data containing multiple diffractors. (a) All 992 automatically picked data points plotted into the zero-offset semblance section. (b) The constant initial model for the inversion containing the initial ray starting locations. (c) The inverted model with the final ray starting locations. (d) The correct velocity model with the position of the diffractor indicated by a black dot.

\section{Bauer, Schwarz \& Gajewski -}




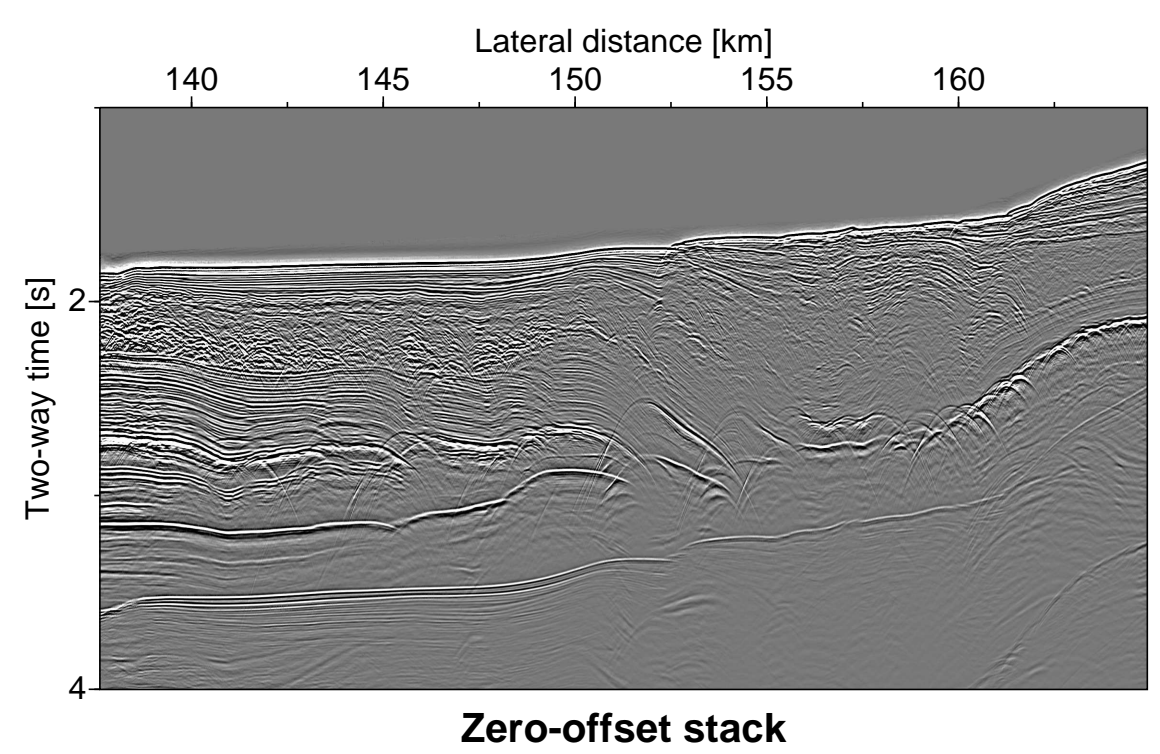

(a)

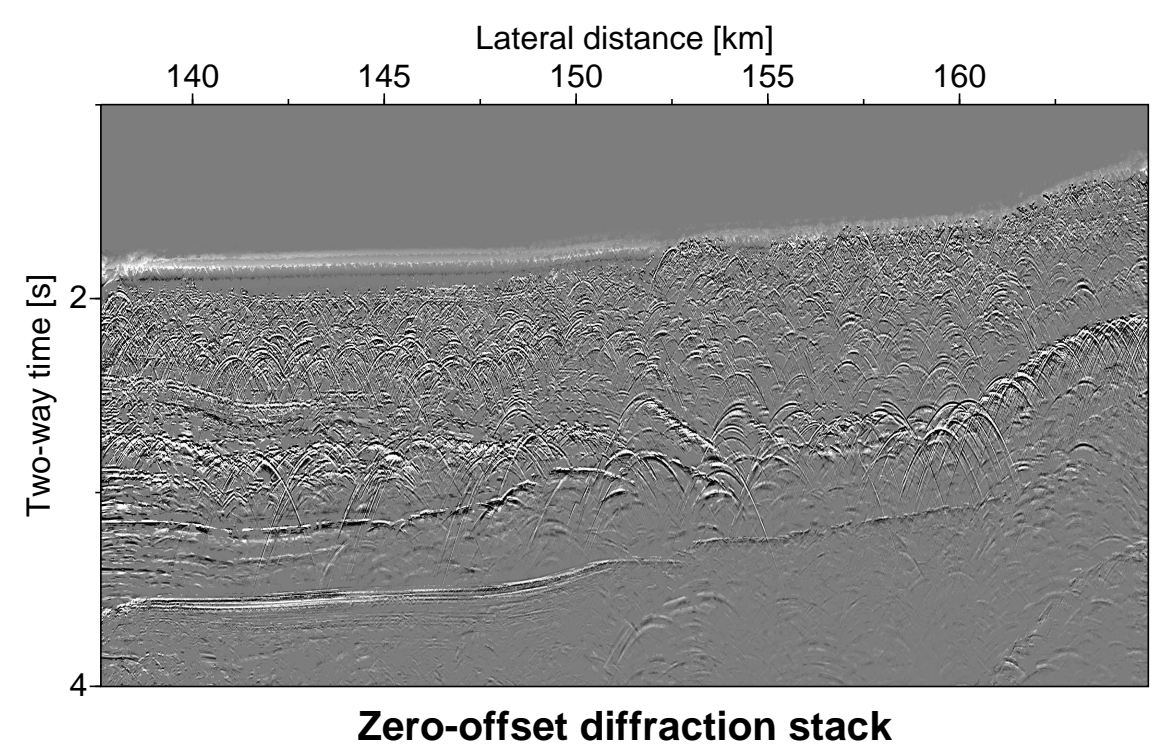

(b)

Figure 5: (a) Zero-offset CRS stack of the investigated marine field data. (b) ZO CRS stack after diffraction separation.

Bauer, Schwarz \& Gajewski - 


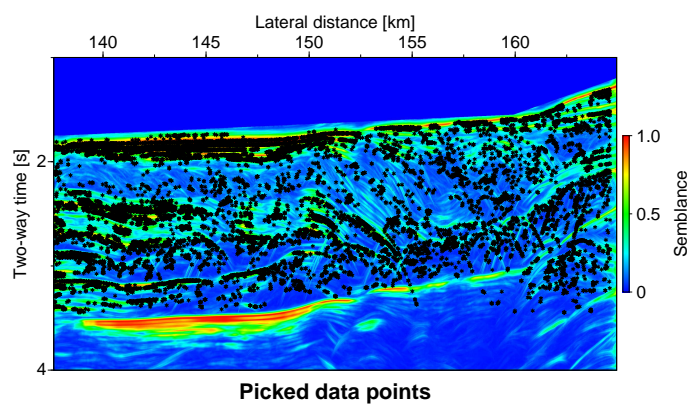

(a)

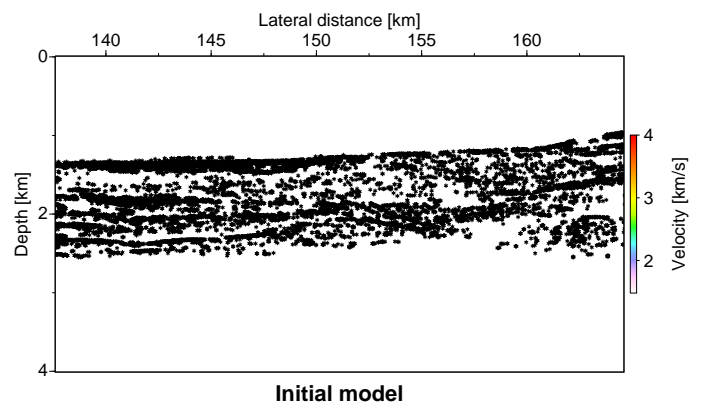

(b)

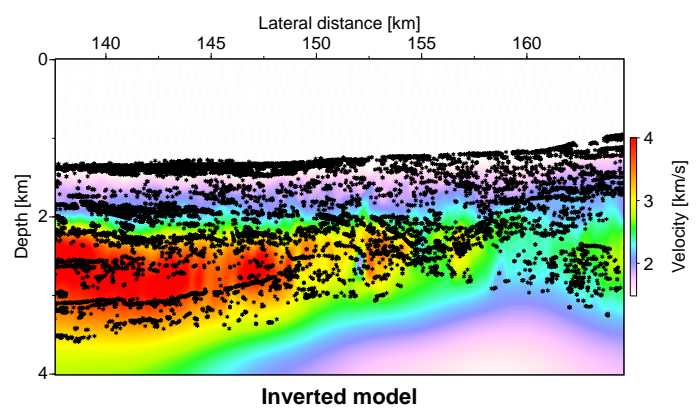

(c)

Figure 6: Field data results. (a) All 11955 automatically picked data points plotted into the zerooffset semblance section. (b) The constant initial model for the inversion containing the initial ray starting locations. (c) Resulting model of the joint inversion using both reflection and diffraction picks overlain with the final ray starting locations.

\section{Bauer, Schwarz \& Gajewski -}




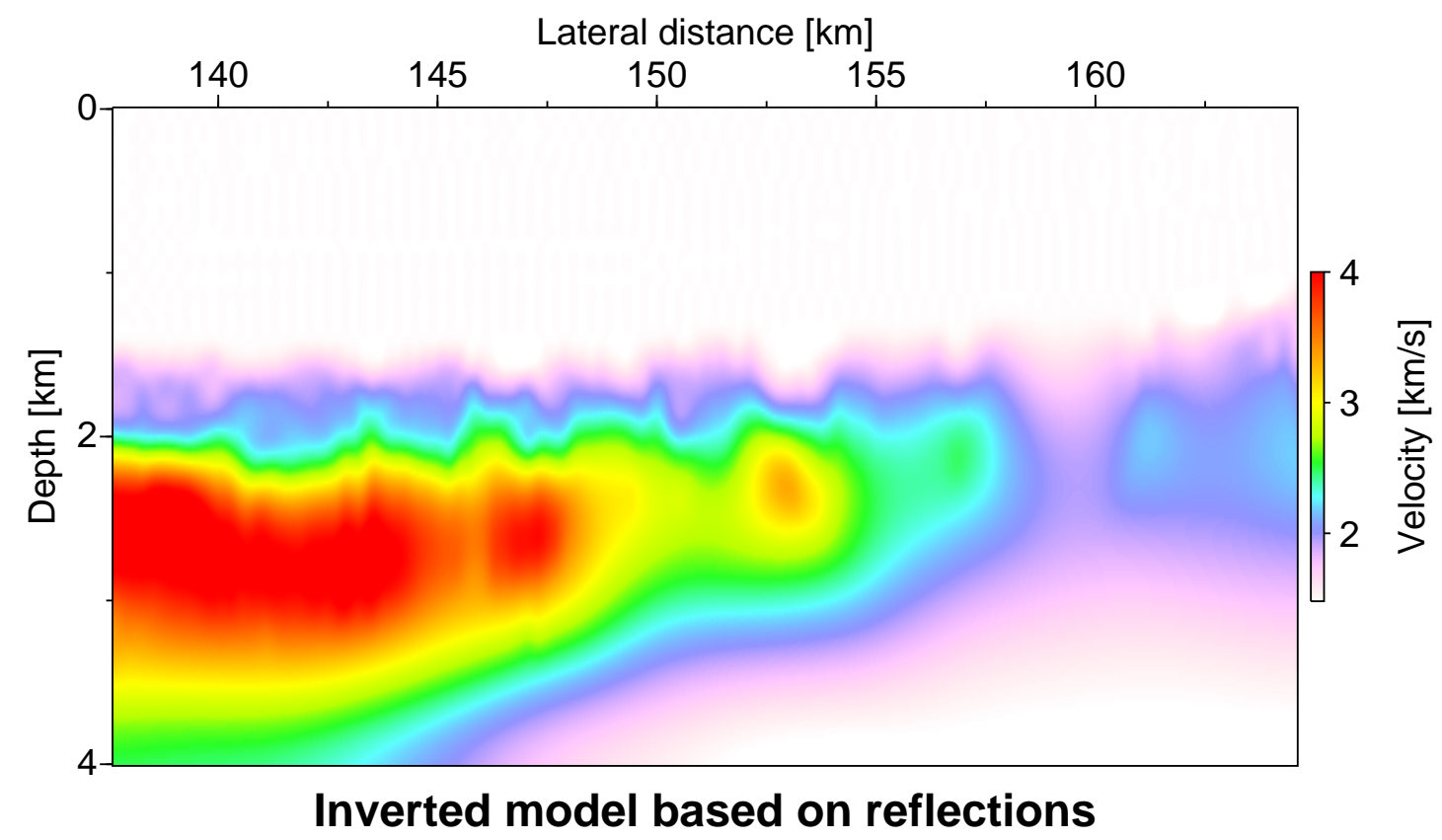

(a)

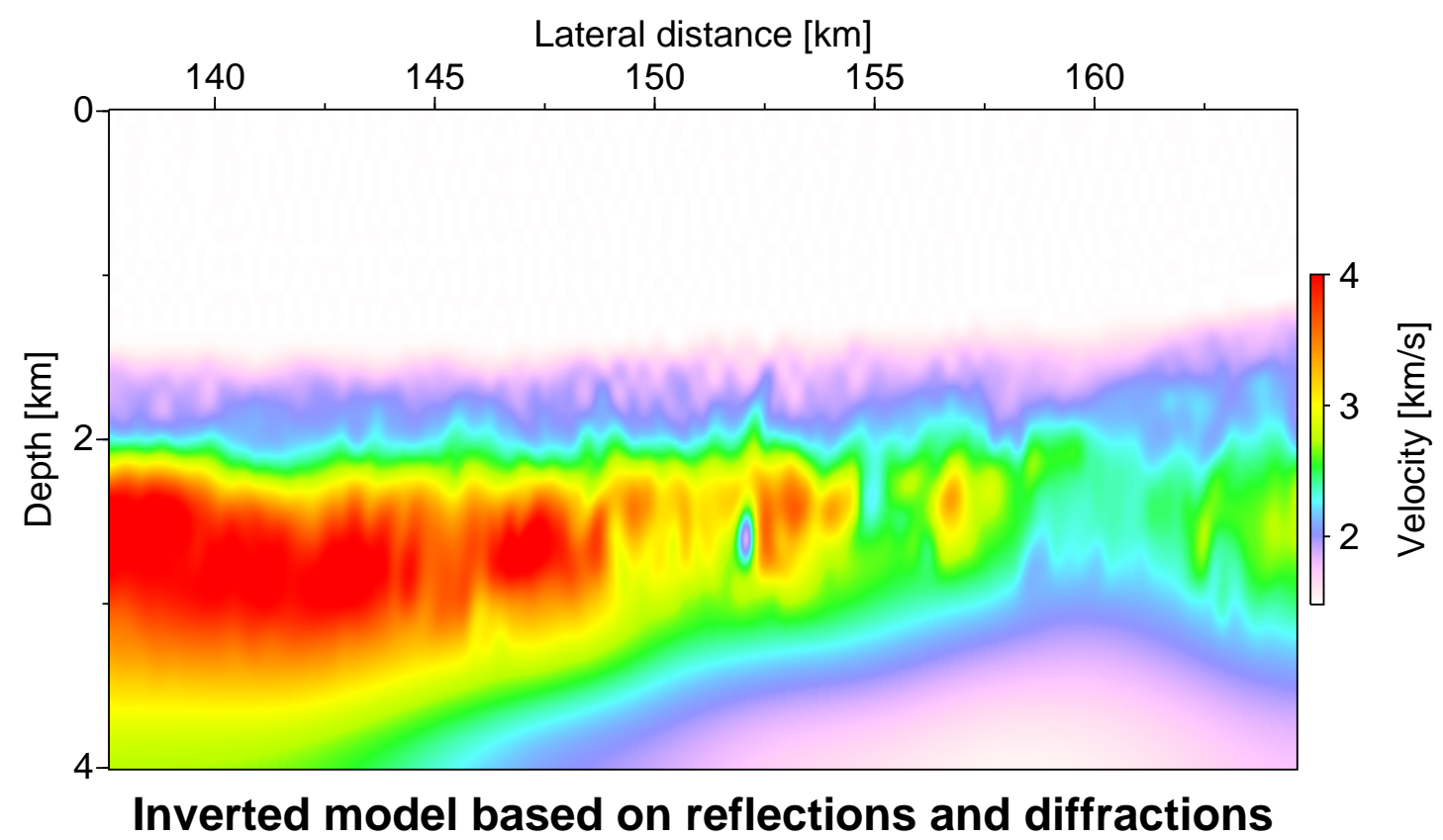

(b)

Figure 7: Velocity models obtained by (a) inversion of only reflections and (b) joint inversion of reflections and diffractions.

Bauer, Schwarz \& Gajewski - 


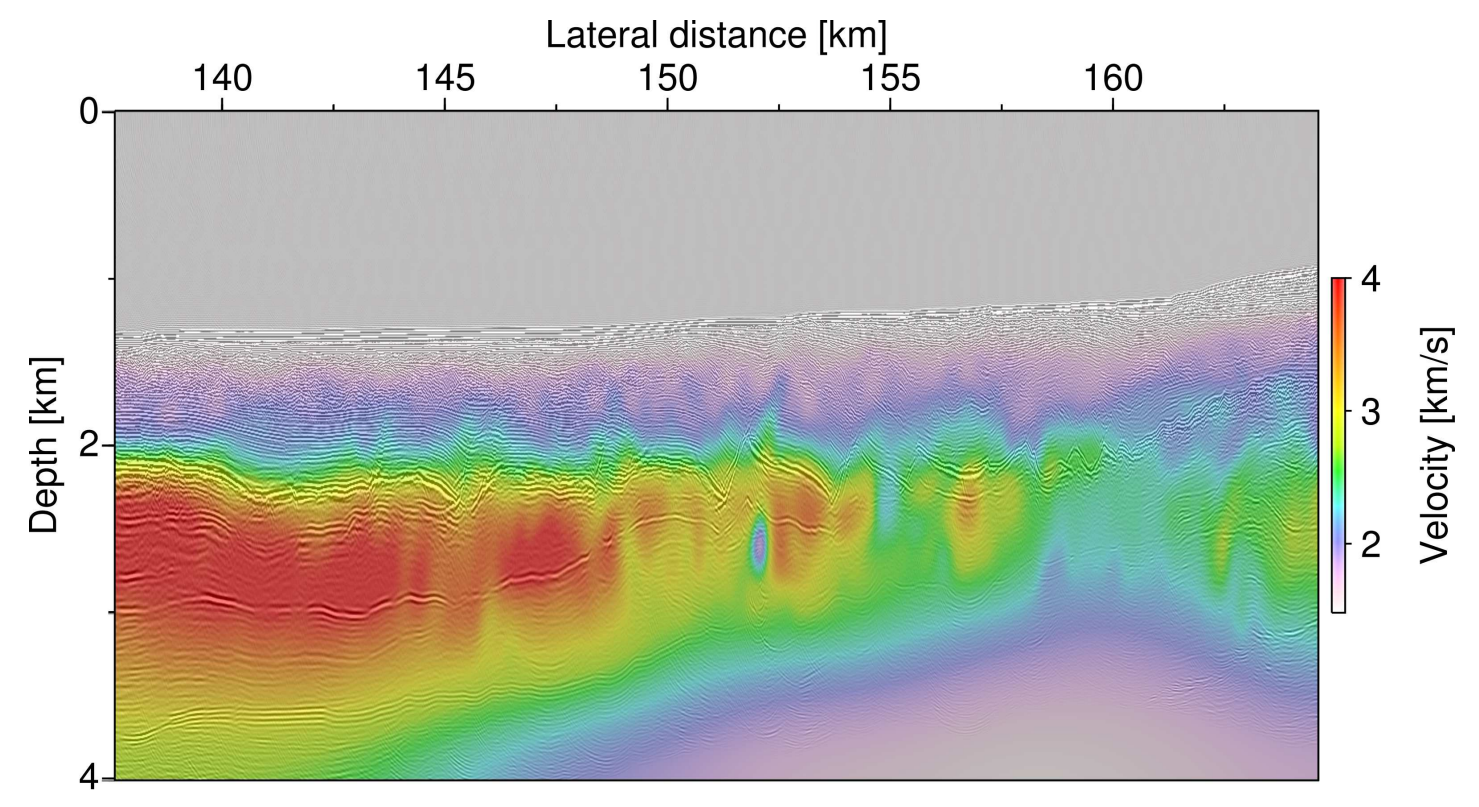

Figure 8: Reverse-time depth-migrated image of the marine field data overlain with the final velocity model obtained from joint inversion of reflections and diffractions.

Bauer, Schwarz \& Gajewski - 


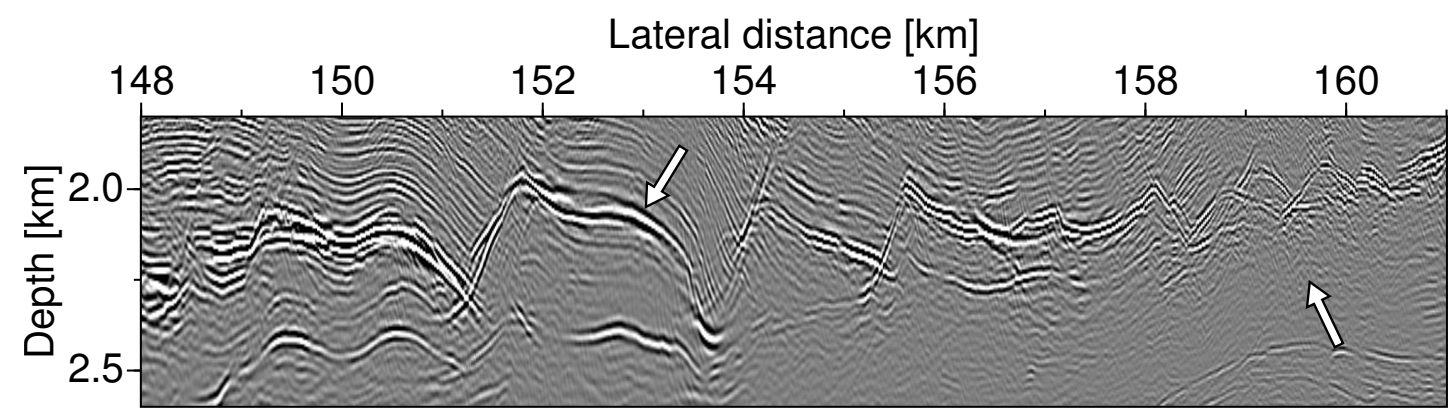

RTM: reflection-only inversion

(a)

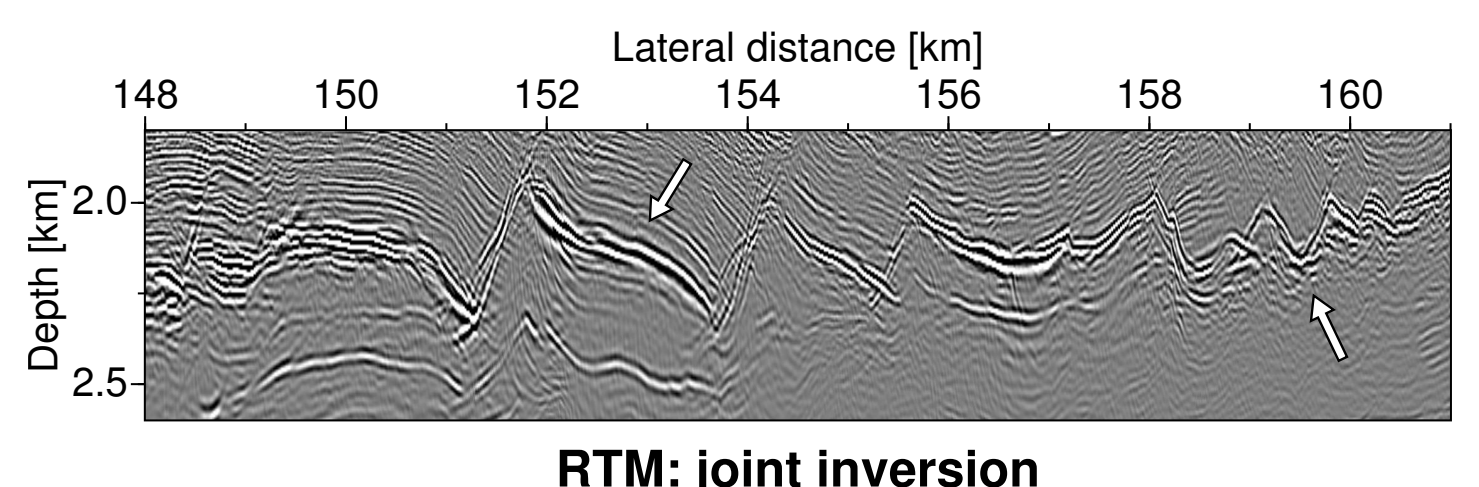

(b)

Figure 9: Excerpts of the reverse-time-migrated images obtained with (a) the reflection-based velocity model and (b) the velocity model from joint inversion of diffractions and reflections. Bauer, Schwarz \& Gajewski - 


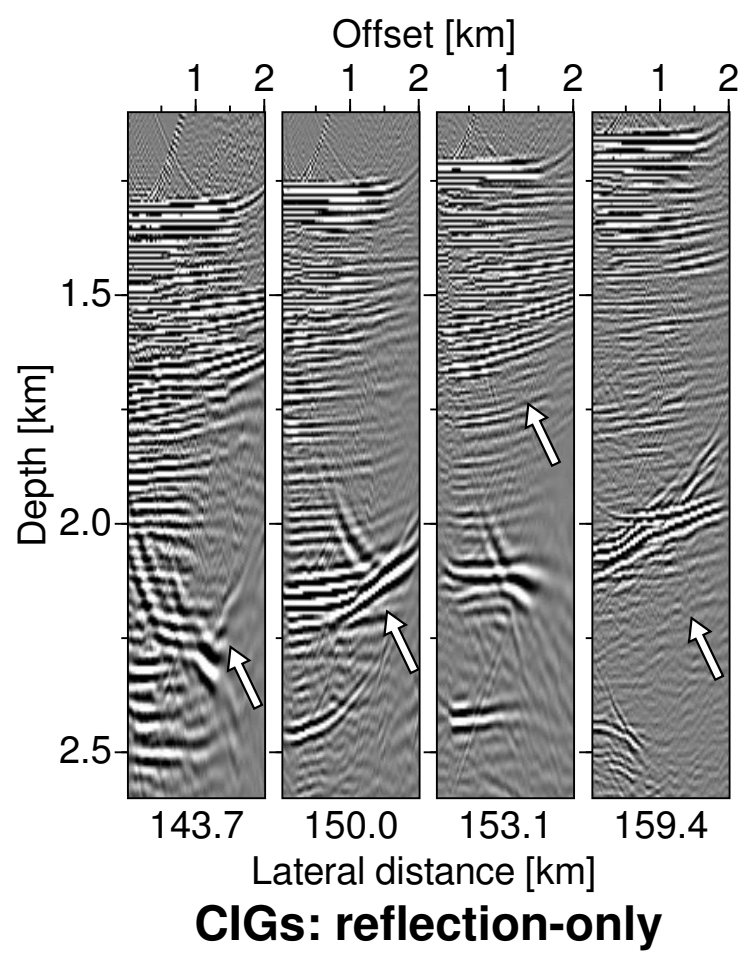

(a)

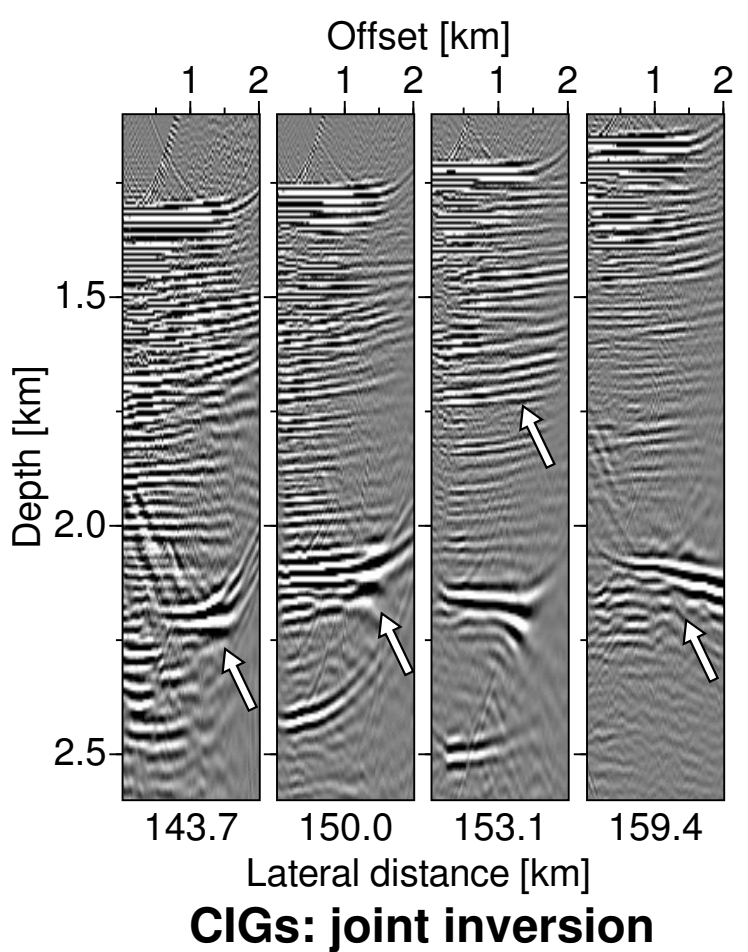

(b)

Figure 10: Common-image-gathers (CIGs) of reverse-time-migrated data obtained with (a) the reflection-based velocity model and (b) the velocity model from joint inversion of diffractions and reflections for four different midpoint locations with a maximum offset of $2 \mathrm{~km}$ each. Bauer, Schwarz \& Gajewski - 